\title{
Stearoyl-CoA desaturase 1 deficiency protects mice from immune-mediated liver injury
}

\author{
Dechun Feng ${ }^{1}$, Ying Wang ${ }^{2}$, Yunhua $\mathrm{Mei}^{2}$, Yan Xu' ${ }^{1}$, Heng Xu ${ }^{1}$ Yun Lu', Qingqiong Luo ${ }^{2}$, Shupei Zhou ${ }^{3}$, \\ Xiangyin Kong ${ }^{1}$ and Lingyun $\mathrm{Xu}^{1,2}$
}

Immunity and metabolism are closely linked. The liver is an important metabolic organ in the body. However, the interactions between hepatocytes and the immune system are poorly understood. In mice developing concanavalin A (ConA)-induced hepatitis $(\mathrm{ClH})$, we found extensive lipid accumulation in hepatocytes. Critical enzyme involved in fat synthesis such as stearoyl-CoA desaturase 1 (SCD1) was upregulated. When we injected ConA to SCD1-deficient mice, we found these mice to be highly resistant to $\mathrm{ClH}$. The mechanisms of the protective effect of SCD1 deficiency might be attributed to the reduced leptin levels in those mice, which modulated critical cytokines and signaling pathways in $\mathrm{CIH}$ pathogenesis. In conclusion, our study suggests that SCD1 deficiency protects mice from liver injury in a leptin-dependent manner.

Laboratory Investigation (2009) 89, 222-230; doi:10.1038/labinvest.2008.105; published online 10 November 2008

KEYWORDS: concanavalin A-induced hepatitis; stearoyl-CoA desaturase 1; lipid accumulation; leptin; STAT1

Accumulated evidence has indicated that immunity and energy metabolism are closely linked. Starvation and malnutrition can suppress immune responses and increase susceptibility to infections, whereas obesity is associated with a state of aberrant immune activity and increased risk for inflammatory diseases. ${ }^{1}$ The liver is the largest and very important metabolic organ in the body. It processes the major categories of nutrients after their absorption from the digestive tract, and stores glycogen, fats and vitamins. In recent years, the role of the liver as a major immune organ has been increasingly recognized. ${ }^{2}$

Immune cells, including Kupffer cells and lymphocytes, constitute approximately $45 \%$ of the total nonhepatocytes cells of a normal liver. ${ }^{3}$ These cells play critical roles in immune defenses against invading pathogens. Mice with highfat diet-induced hepatic steatosis show decreased natural killer T-cell (NKT cell) proportions in the liver, and are sensitive to lipopolysaccharide (LPS)-induced liver injury. ${ }^{4,5}$ Moreover, in hepatosteatotic mice developing concanavalin A
(ConA)-induced hepatitis (CIH), T-cell differentiation is strongly shifted toward a Th1 profile. In addition, severe liver injury and high production of proinflammatory cytokines, including tumor necrosis factor- $\alpha(\mathrm{TNF}-\alpha)$ and interferon- $\gamma$ $($ IFN- $\gamma)$, have been observed in hepatosteatotic mice. ${ }^{6}$ Therefore, metabolic syndromes have been proposed as risk factors in immune-mediated liver inflammation and injury. ${ }^{5}$ However, the manner in which inflammation influences lipid metabolism in the liver and the interactions between immune cells and hepatocytes are still poorly understood.

In our study with $\mathrm{CIH}$ in mice, we noticed extensive lipid accumulation in hepatocytes. The critical enzyme involved in fat synthesis, stearoyl-CoA desaturase 1 (SCD1), was greatly upregulated. In attempting to induce CIH in SCD1-deficient mice, we found these mice to be highly resistant to $\mathrm{CIH}$. The mechanisms leading to $\mathrm{CIH}$ resistance in SCD1-deficient mice were then elucidated. Our results add another line of direct evidence that hepatosteatosis greatly influences immune responses in the liver. Thus, we suggest that

\footnotetext{
${ }^{1}$ Institute of Health Sciences, Shanghai Institutes for Biological Sciences, Chinese Academy of Sciences, Shanghai Jiaotong University School of Medicine, Shanghai, China; ${ }^{2}$ Shanghai Institute of Immunology, Shanghai Jiaotong University School of Medicine, Shanghai, China and ${ }^{3}$ Department of Laboratory Animal Science, Health Science Center, Peking University, Beijing, China

Correspondence: Professor L Xu, Shanghai Institute of Immunology, Shanghai Jiaotong University, 225 South Chongqing Road, Building I Room 308, Shanghai 200025, China.
}

E-mail: lyxu@sibs.ac.cn

Received 19 June 2008; revised 29 September 2008; accepted 2 October 2008 
modulation of fat metabolism be further explored as a potential strategy for intervention and treatment of inflammatory liver diseases.

\section{MATERIALS AND METHODS Animal Treatment}

C57BL/6 male mice, 8-10 weeks of age, were purchased from Shanghai SLAC Laboratory Animal CO LTD (Shanghai, China). $a b^{\mathrm{Xyk}}$ mice (back crossed to Balb/c mice, F2 generation) with the spontaneous dysfunction of SCD1 have been described previously. ${ }^{7}$ Mice were housed in the animal facilities of Shanghai Institutes for Biological Science, Chinese Academy of Sciences, under pathogen-free conditions according to the Institutional Animal Care and Use Committee guidelines. Mice were fed ad libitum a standard laboratory chow diet provided by SLAC Laboratory Animal CO LTD.

\section{$\mathrm{ClH}$ Induction}

Mice were injected via the tail vein with a single dose $(15 \mathrm{mg} / \mathrm{kg}$ of body weight) of ConA to produce the $\mathrm{CIH}$ model.

\section{Leptin Replacement}

$\mathrm{ab}^{\mathrm{Xyk}} / \mathrm{ab}^{\text {Xyk }}$ mice received $1 \mathrm{mg} / \mathrm{kg}$ recombinant murine leptin (R\&D Systems, MN, USA) or PBS by intraperitoneally injection twice a day for 5 days. Subsequently, ConA was injected into all the mice.

\section{Alanine Aminotransferase Measurement}

Plasma was obtained approximately $17 \mathrm{~h}$ after ConA injection. Alanine aminotransferase (ALT) levels were determined using the ALT detection kit (Shanghai Yihua Medical Science \& Technology, Shanghai, China) according to manufacturer's instructions.

\section{Histology}

Livers were removed after perfusion with PBS, fixed with $4 \%$ phosphate-buffered paraformaldehyde, and embedded in paraffin. Tissue sections $(5 \mu \mathrm{m})$ were prepared, stained with Haematoxylin \& Eosin (H\&E), and examined under light microscopy. A total of 10 tissue sections were analyzed for each animal.

\section{TUNEL Staining}

Paraffin-embedded liver tissues were assayed for DNA fragmentation using a terminal deoxynucleotidyl transferasemediated dUTP-biotin nick end labeling (TUNEL) reaction according to the manufacturer's instructions (Roche Molecular Biochemicals, IN, USA). Sections were then examined under light microscopy. A total of 10 tissue sections were analyzed for each animal.

\section{Oil Red O Staining}

Frozen liver sections $(8 \mu \mathrm{m})$ were stained with Oil Red O (Sigma, MO, USA) for $10 \mathrm{~min}$, and then counterstained with hematoxylin for $45 \mathrm{~s}$. Sections were then examined under light microscopy. A total of 10 tissue sections were analyzed for each animal.

\section{Analysis of Plasma Cytokines}

Plasma concentrations of TNF- $\alpha$, IFN- $\gamma$ and leptin were determined using specific enzyme-linked immunosorbent assay kits (R\&D Systems) according to the manufacturer's instructions.

\section{RNA Extraction and Reverse Transcription}

Total RNA was isolated from cell pellets and liver tissues using the RNeasy Mini Kit (Qiagen, Hilden, Germany). Genomic DNA was removed from total RNA before cDNA synthesis using the RNase-free DNase set for DNase digestion during RNA purification (Qiagen). RNA was stored at $-80^{\circ} \mathrm{C}$. First-strand cDNA synthesis was performed for each RNA sample using Sensiscript RT Kit (Qiagen). Random hexamers were used to prime cDNA synthesis.

\section{Real-Time PCR}

Gene expression of inducible nitric oxide synthase (iNOS), interferon-induced protein 10 (IP-10), monocyte chemotactic protein-1 (MCP-1), macrophage inflammatory protein- $1 \alpha$ (MIP-1 $\alpha$ ), SCD1 and leptin mRNA was performed by realtime PCR using SYBR Green master mix (Applied Biosystems, Foster City, CA, USA). Thermocycler conditions included an initial holding period at $50^{\circ} \mathrm{C}$ for $2 \mathrm{~min}$, then $95^{\circ} \mathrm{C}$ for $10 \mathrm{~min}$. This was followed by a two-step PCR program consisting of $95^{\circ} \mathrm{C}$ for $15 \mathrm{~s}$, and $60^{\circ} \mathrm{C}$ for $60 \mathrm{~s}$ for 40 cycles. Data were collected and quantitatively analyzed using an ABI Prism 7900 sequence detection system (Applied Biosystems). The $\beta$-actin was used as an endogenous control to normalize for differences in the amount of total RNA in each sample. All quantities were expressed as number of fold relative to the expression of $\beta$-actin.

$\beta$-actin:

sense 5'-TGTCCACCTTCCAGCAGATGT-3'; anti-sense $5^{\prime}$-AGCTCAGTAACAGTCCGCCTAGA- ${ }^{\prime}$. MIP- $1 \alpha$ :

sense $5^{\prime}$-CACCCTCTGTCACCTGCTCAA-3'; anti-sense $5^{\prime}$-ATGGCGCTGAGAAGACTTGGT- ${ }^{\prime}$. IP-10: sense 5'-GCCGTCATTTTCTGCCTC-3'; anti-sense $5^{\prime}$-ATGGCGCTGAGAAGACTTGGT- ${ }^{\prime}$. iNOS:

sense $5^{\prime}$-GCCACCAACAATGGCAACA-3'; anti-sense $5^{\prime}$-CGTACCGGATGAGCTGTGAATT- 3 ' MCP-1:

sense 5'-AAAAACCTGGATCGGAACCAA-3'; anti-sense $5^{\prime}$-CGGGTCAACTTCACATTCAAAG- ${ }^{\prime}$. Leptin:

sense 5'-TGCACCCTATGTCACCATCAAA-3'; anti-sense $5^{\prime}$-CAGAGCTGAGCACGAAACTGTG-3' 
a

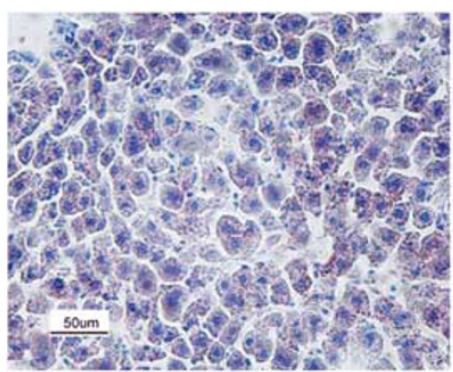

$8 \mathrm{~h}$

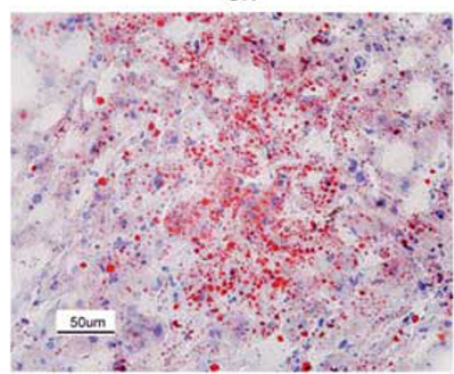

$2 \mathrm{~h}$

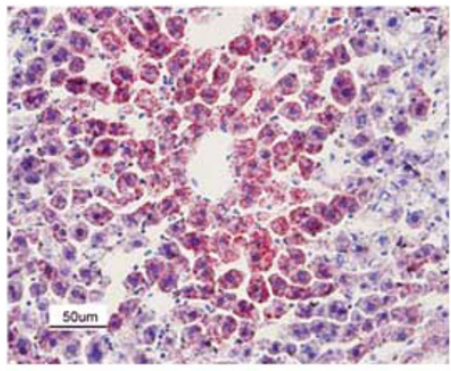

$24 \mathrm{~h}$

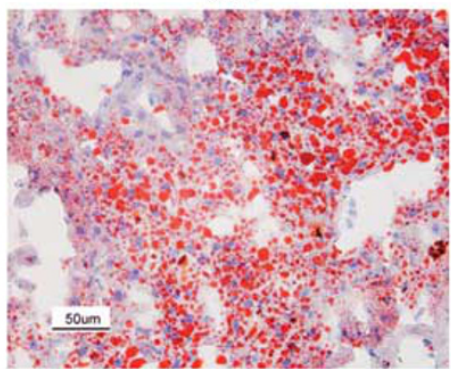

b
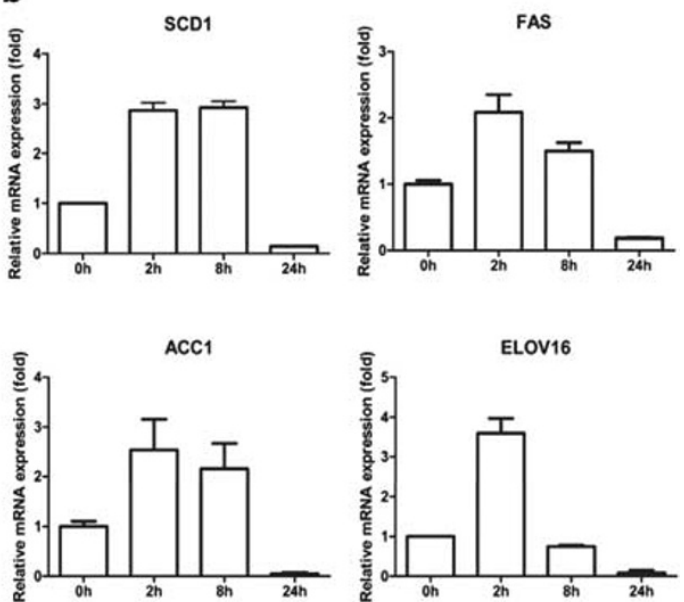

Figure 1 Lipid accumulation in CIH. Four groups of C57BL/6 mice $(n=5)$ received a ConA $(15 \mathrm{mg} / \mathrm{kg})$ injection via the tail vein. (a) Representative photomicrographs are shown depicting Oil Red O staining of liver sections. Increased fat (red) in hepatocytes 2-24 h after ConA injection as compared to a quiescent condition $(0 \mathrm{~h})$. (b) Liver mRNA expression of SCD1, FAS, ACC1 and Elov16. Results are shown as mean \pm s.d. Data are representative of five experiments.

SCD1:

sense $5^{\prime}$-AAGTTCTCAGAAACACACGCCG-3'; anti-sense $5^{\prime}$-TTCCGCCCTTCTCTTTGACAG-3'

\section{Immunoblot Analysis}

Immunoblot analysis were performed using whole liver cell extract samples resolved by electrophoresis on a $10 \%$ SDS-polyacrylamide gel and transferred to PVDF membrane. STAT1, phospho-STAT1 (Thr-701), STAT3 and phosphoSTAT3 (Tyr-705) were visualized using antibody from BD Bioscience (CA, USA).

\section{Electrophoretic Mobility Shift Assay}

Nuclear extracts of liver tissues were prepared as described previously. ${ }^{8} \quad \mathrm{NF}-\kappa \mathrm{B}$-binding consensus single-strand oligo nucleotide (5'-AGTTGAGGGGACTTTCCCAGGC-3') was first annealed with the complement oligonucleotide (5'-GCCTGGGAAAGTCCCTCAACT- $\left.3^{\prime}\right)$. The annealed DNA fragment was labeled with $\left[\gamma_{-}{ }^{32} \mathrm{P}\right]$ dATP (Amersham, Piscataway, NJ, USA) using T4 Polynucleotide Kinase (Promega, Madison, WI, USA). Nuclear proteins $(15 \mu \mathrm{g})$ were incubated with $2.5 \mathrm{ng}{ }^{32} \mathrm{P}$-labeled double-stranded oligonucleotide probes for $30 \mathrm{~min}$ at room temperature. The mixture was resolved by electrophoresis on $4 \%$ polyacrylamide gels with $0.5 \times$ Tris-borate-ethylenediaminetetraacetic acid buffer at $4^{\circ} \mathrm{C}$.

\section{Statistical Analysis}

All the results were expressed as mean \pm s.d. Statistical comparisons between two groups were made using Student's $t$-test after analysis of variance. The level of significance was set at $\alpha=0.05$. All the tests were two-sided.

\section{RESULTS}

ConA Treatment Increases Fat Accumulation in the Liver $\mathrm{CIH}$ is a widely used animal model of immune-mediated liver injury and can be induced by intravenous injection of ConA into mice. We found that as early as $2 \mathrm{~h}$ after ConA injection, fat accumulation was observed in hepatocytes as indicated by Oil Red $\mathrm{O}$ staining (Figure 1a). Meanwhile, the critical enzyme involved in fat synthesis, SCD1, was upregulated (Figure 1b). We also found that other key lipogenic genes including fatty acid synthase, acetyl-CoA carboxylase 1 and Elovl6 showed a similar expression pattern with SCD1 (Figure 1b). Thus, at the early phase of $\mathrm{CIH}$ induction, in tandem with the development of tissue damaging immune responses, there arose increased fat synthesis and accumulation.

\section{Deficiency of SCD1 Protects Mice from CIH}

SCD1 plays a crucial role in lipid metabolism. ${ }^{9,10}$ SCD1deficient mice manifest defective hepatic cholesterol ester and triglyceride synthesis, ${ }^{11,12}$ thus exhibiting reduced liver steatosis. The $\mathrm{ab}^{\mathrm{Xyk}}$ mice $^{7}$ are newly characterized asebia mice with a spontaneous dysfunction mutation of the SCD1 gene. We used these mice for induction of $\mathrm{CIH}$. Their littermates 
a

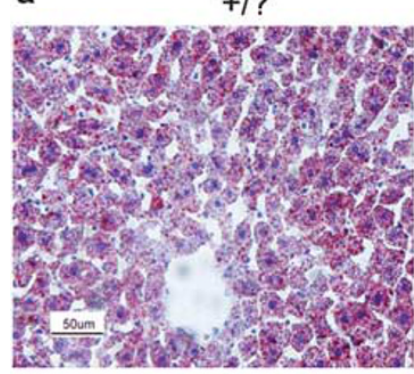

C

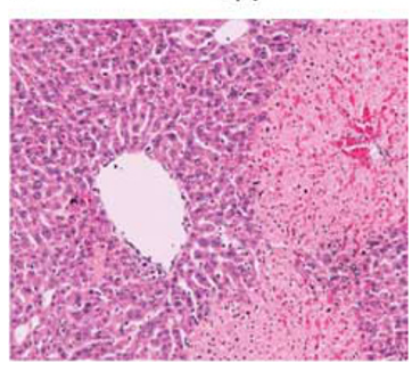

$a b^{X y k} / a b^{X y k}$
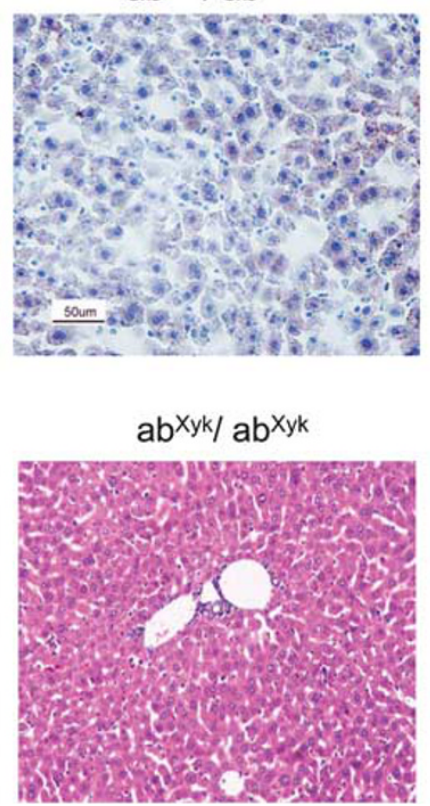

b

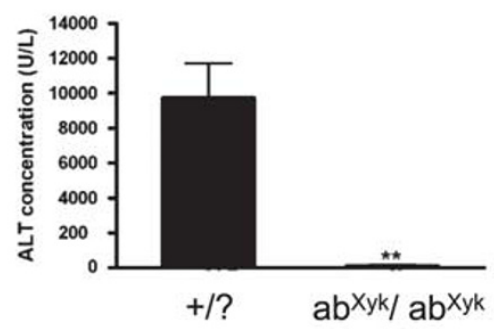

$+/ ?$

d

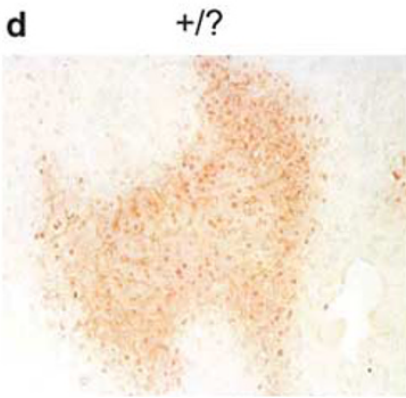

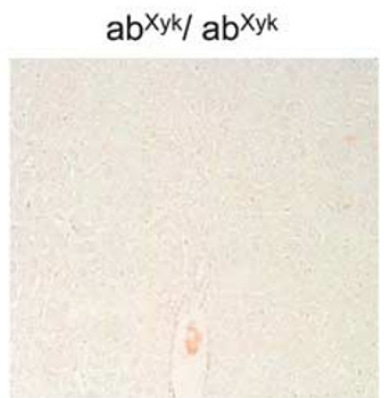

Figure 2 SCD1-deficient mice are resistant to ConA-induced hepatitis. SCD1-deficient mice $\left(a b^{\mathrm{Xyk}} / \mathrm{bb}^{\mathrm{Xyk}}\right)$ and littermate control mice $(+/$ ? $)(n=5)$ received a ConA injection $(15 \mathrm{mg} / \mathrm{kg})$ via the tail vein. Oil Red O staining of liver sections $4 \mathrm{~h}$ after ConA injection (a). Seventeen hours after ConA injection, plasma was obtained and ALT levels were measured (b). At the same time, livers were removed and fixed in $4 \%$ paraformaldehyde. Liver H\&E (c) and TUNEL (d) staining were performed and results examined using light microscopy (magnification $\times 200$ ). Results are shown as mean \pm s.d. Data are representative of five experiments. ${ }^{* *} P<0.01$.

with normal phenotype $\left(+/+\right.$ or $+/ \mathrm{ab}^{\mathrm{Xyk}}$ mice, shown as $+/$ ?) were used as controls.

Four hours after $\mathrm{CIH}$ induction, significantly less hepatosteatosis was observed in $a b^{\mathrm{Xyk}} / \mathrm{ab}{ }^{\mathrm{Xyk}}$ mice as compared with $+/$ ? mice (Figure 2a). Serum was collected $17 \mathrm{~h}$ after ConA administration for the measurement of ALT to verify liver damage severity. We found that serum ALT was dramatically increased after $\mathrm{CIH}$ induction in $+/$ ? mice $(9183 \pm 1629, \mathrm{U} / \mathrm{L})$, indicating severe liver damage in these mice. However, in $a b^{X y k} / a b^{X y k}$ mice, ConA-induced serum ALT increase was markedly blocked $(88 \pm 50, \mathrm{U} / \mathrm{L} ; P<0.01)$ (Figure 2b). Microscopic examination of liver sections from $+/$ ? mice with $\mathrm{CIH}$ revealed severe pathological changes, characterized by widespread inflammatory lesions (Figure $2 \mathrm{c}$, $\mathrm{H} \& \mathrm{E}$ staining) and hepatocyte death (Figure 2d, TUNEL staining). In contrast, few inflammatory or necrotic lesions were observed in $a b^{\mathrm{Xyk}} / \mathrm{ab}^{\mathrm{Xyk}}$ mice (Figure $2 \mathrm{c}$ and $\mathrm{d}$ ). Therefore, mice deficient in SCD1 appeared highly resistant to $\mathrm{CIH}$.

\section{SCD1 Deficiency Suppresses Inflammatory Molecules in CIH Pathogenesis}

Levels of serum TNF- $\alpha$ and IFN- $\gamma$, which are crucial in the pathogenesis of $\mathrm{CIH}$, were measured as well. Compared with those in $+/$ ? mice, the increases in TNF- $\alpha$ and IFN- $\gamma$ production were greatly attenuated in $a b^{X y k} / a b^{X y k}$ mice (Figure $3 \mathrm{a}$ and $\mathrm{b}$ ). The expression of several major inflammatory mediators including iNOS, IP-10, MCP-1, MIP$1 \alpha$, which were reported to be important in $\mathrm{CIH}$ pathogenesis, was also examined. Expression of all these inflammatory mediators was found significantly lower in $a b^{X y k} / a b^{X y k}$ mice than in $+/$ ? mice after $\mathrm{CIH}$ induction (Figure $3 \mathrm{c}-\mathrm{f}$ ).

\section{SCD1 Deficiency Suppresses NF- $\kappa$ B and STAT1 Transcriptional Pathways}

Alterations of molecular pathways involved in $\mathrm{CIH}$ pathogenesis were then determined. First, NF- $\kappa \mathrm{B}$ activity was measured by electrophoretic mobility shift assay (EMSA). We found that DNA-binding activity of NF- $\kappa \mathrm{B}$ was strongly reduced in $a b^{X y k} / a b^{X y k}$ mice as compared with that in $+/$ ? mice (Figure 4a). Next, we determined phosphorylated and nonphosphorylated STAT1 and STAT3 levels by immunoblot analysis. The protein levels of STAT1 in the liver were lower in $a b^{X y k} / a b^{X y k}$ mice than in $+/$ ? mice. At the same time, phosphorylation of STAT1 was also suppressed in $a b^{X y k} / a b^{X y k}$ mice. No significant difference of total protein levels and the phosphorylated form of STAT3 were observed in $+/$ ? and $\mathrm{ab}^{\mathrm{Xyk}} / \mathrm{ab}^{\mathrm{Xyk}}$ mice with CIH (Figure $4 \mathrm{~b}$ ). These results indicate that reduced proinflammatory cytokine production and impaired pathogenic signaling pathways in $a b^{\mathrm{Xyk}} / \mathrm{ab}{ }^{\mathrm{Xyk}}$ mice made these mice resistant to liver damage induced by ConA challenge.

\section{Reduced Leptin Levels Mediate Resistance to $\mathrm{CIH}$ in SCD1-Deficient Mice}

Leptin is a well-characterized adipokine and is considered as a proinflammatory cytokine which mediates the crosstalk between metabolism and immunity. ${ }^{13}$ Previous studies have 

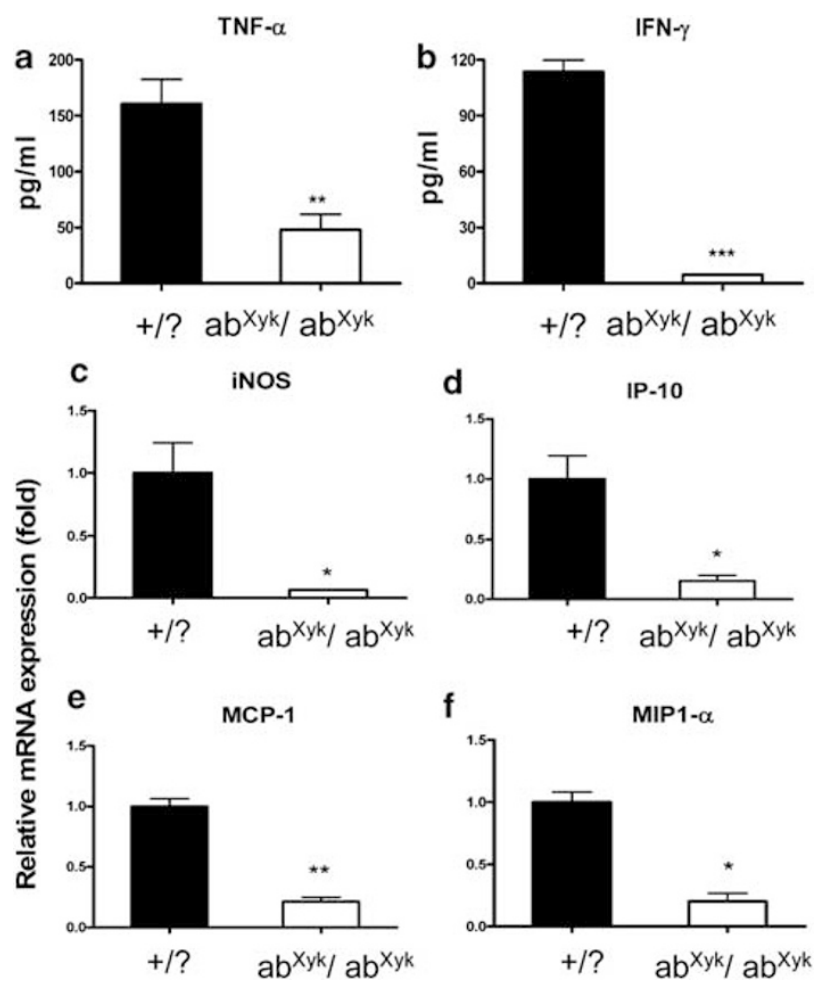

d

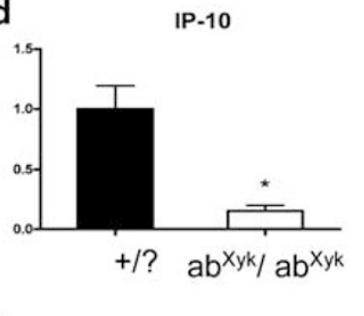

f

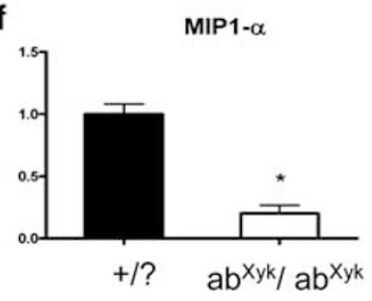

Figure 3 SCD1-deficient mice with $\mathrm{CIH}$ produced less inflammatory molecules. SCD1-deficient mice $\left(a b^{\mathrm{xyk}} / a b^{\mathrm{xyk}}\right)$ and littermate control mice $(+/ ?)(n=5)$ received a ConA injection $(15 \mathrm{mg} / \mathrm{kg})$ via the tail vein. Plasma TNF- $\alpha$ levels (a) $2 \mathrm{~h}$ after ConA injection and IFN- $\gamma$ levels (b) $8 \mathrm{~h}$ after ConA injection were measured using ELISA. Liver mRNA expressions of iNOS (c), IP-10 (d), MCP-1 (e) and MIP-1 $\alpha$ (f) were quantitatively analyzed using realtime PCR. Results are shown as mean \pm s.d. Data are representative of five experiments. ${ }^{*} P<0.05,{ }^{* *} P<0.01,{ }^{* * *} P<0.001$.

shown significantly reduced leptin levels in SCD1-deficient mice on chow and high-fat diet. ${ }^{14}$ Moreover, mice deficient in leptin are resistant to $\mathrm{CIH}$ induction. ${ }^{15}$ Therefore, we investigated whether leptin was involved in the resistance to $\mathrm{CIH}$ in $\mathrm{ab}^{\mathrm{Xyk}} / \mathrm{ab}^{\mathrm{Xyk}}$ mice. First, we examined leptin mRNA expression in adipose tissues, as well as its protein concentration in serum in $\mathrm{ab}^{\mathrm{Xyk}} / \mathrm{ab}^{\mathrm{Xyk}}$ mice. Consistent with previous studies, both mRNA and protein levels of leptin were decreased significantly in these mice (Figure 5a). Leptin replacement experiments were then carried out. $\mathrm{ab}^{\mathrm{Xyk}} / \mathrm{ab}^{\mathrm{Xyk}}$ mice received recombinant murine leptin $(1 \mathrm{mg} / \mathrm{kg})$ or PBS injection twice a day for 5 days, and $\mathrm{CIH}$ was subsequently induced in all the mice. As a result, leptin administration significantly restored the ability of ConA to induce hepatotoxicity in $a b^{X y k} / a^{X y k}$ mice (Figure $5 b$ ). There was small areas of centrilobular necrosis in leptin-treated SCD1-deficient mice with $\mathrm{CIH}$, whereas no necrosis was observed in PBS-treated SCD1-deficient CIH mice (Figure 5c). We also measured inflammatory mediators expression in the liver. The levels of IFN- $\gamma$, IP-10, MCP-1, MIP- $1 \alpha$ were significantly higher in leptin-treated SCD1-deficient $\mathrm{CIH}$ mice (Figure 5d). Therefore, leptin, at least partially, mediated the resistance to $\mathrm{CIH}$ in SCD1-deficient mice.

\section{DISCUSSION}

This study was prompted by our observation of extensive lipid accumulation in the livers of $\mathrm{CIH}$ mice. Previous studies have pointed out the connection between hepatic steatosis and liver inflammatory damage. NKT cells are selectively reduced in fatty liver of diet-induced obesity mice, promoting Th1 polarization of and sensitization to LPS-induced liver injury. ${ }^{4,5}$ A similar phenomenon observed in hepatosteatotic mice induced with $\mathrm{CIH}$ favored a Th1 response. Enhanced liver injury was found in these mice. ${ }^{6}$ A newly published study suggests that the loss of regulatory $\mathrm{T}$ cells in fatty liver may be one reason for enhanced inflammatory damage in fatty liver. ${ }^{16}$ Moreover, chronic infectious liver diseases caused by viruses, including $\mathrm{HBV}$ and $\mathrm{HCV}$, are frequently associated with hepatic steatosis. ${ }^{17}$ Severe hepatic steatosis has also been seen in various animal models of liver injury, such as those induced by LPS, ${ }^{18}$ alcohol $^{19}$ and CCl4 (our unpublished data). Because hepatic steatosis presents frequently as a condition leading to liver inflammation, fibrosis and cancer, it would appear that interference with lipid metabolism may indeed represent a useful and novel strategy for prevention and treatment of liver disease.

Along with lipid accumulation, we found enhanced SCD1 expression in the liver of $\mathrm{CIH}$ mice. SCD1 is a central lipogenic enzyme converting saturated long-chain fatty acids into monounsaturated fatty acids (MUFAs). ${ }^{20}$ Then we asked how $\mathrm{CIH}$ would manifest in SCD1-deficient mice. As expected, SCD1-deficient mice were highly resistant to $\mathrm{CIH}$ as indicated by serum ALT measurement and liver histological examination. Although a recently published report showed that SCD1-deficient mice showed increased severity in a dextran sulfate sodium-induced acute colitis model. ${ }^{21}$ The influence of SCD1 deficiency on immune-mediated liver injury was not known. Our findings indicate that SCD1, a key enzyme involved in fat metabolism, is involved in immune-mediated liver injury. We then went further to explore the underlying mechanism.

Immune activation elicited by ConA results in the elevation of various plasma cytokines, which then leads to immune assault upon hepatocytes. TNF- $\alpha$ is secreted from liver macrophages (Kupffer cells) as early as $2 \mathrm{~h}$ after $\mathrm{CIH}$ induction. ${ }^{22}$ Pretreatment of mice with anti-mouse TNF- $\alpha$ anti-serum or TNF- $\alpha$ inhibitor protected them from $\mathrm{CIH} .{ }^{23}$ In addition, depletion of IFN- $\gamma$ resulted in a marked reduction in ConA-induced liver injury and inflammation. ${ }^{24,25}$ IFN- $\gamma$ can synergize with TNF- $\alpha$ to induce production of several chemokines and adhesion molecules. ${ }^{26}$ In $\mathrm{CIH}$ induced mice, the release of $\mathrm{NO}$ into plasma and the expression of iNOS mRNA in liver was found to be increased. ${ }^{24,27}$ The involvement of the toxic effect of iNOS-derived NO in the development of $\mathrm{CIH}$ has been emphasized recently. ${ }^{28,29} \mathrm{NO}$ mediates tissue injury through pathways including inhibition of mitochondrial respiration, inactivation of proteinase inhibitors and formation of free radicals. ${ }^{30}$ During the first hours after challenge, the production of proinflammatory 
a
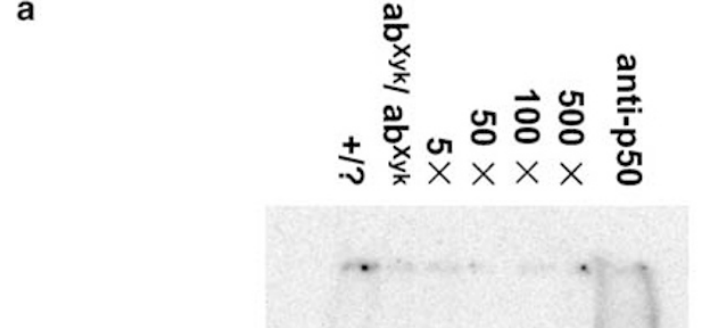

Supershift $\longrightarrow$

Free probe b

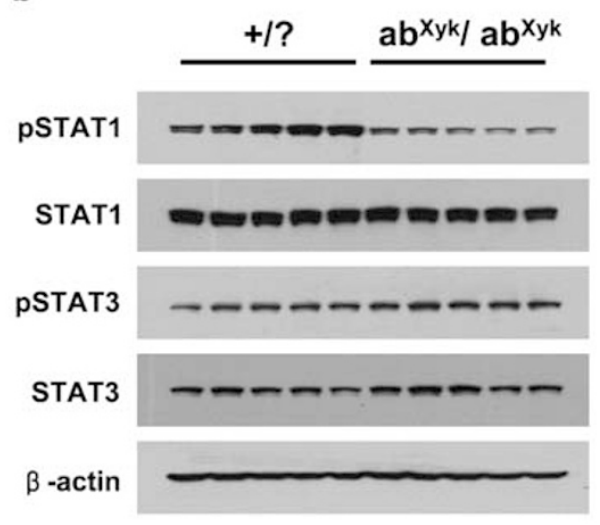

C

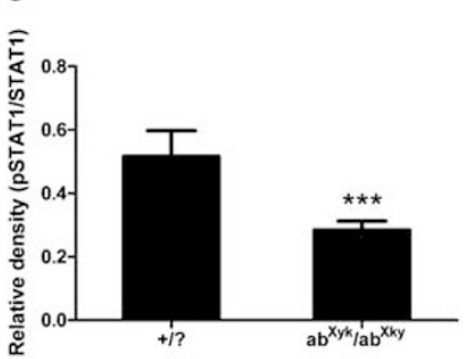

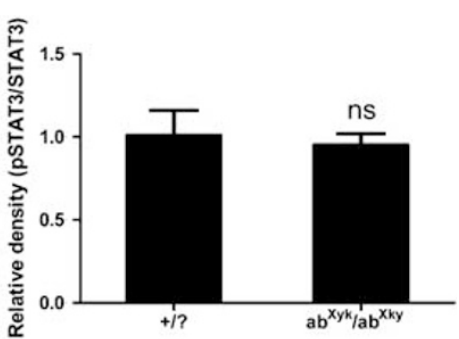

Figure 4 Transcriptional pathways. SCD1-deficient mice $\left(a b^{x y k} / a b^{x y k}\right)$ and littermate control mice $(+/ ?)(n=5)$ received a ConA injection $(15 \mathrm{mg} / \mathrm{kg})$ via the tail vein. (a) Liver nuclear extracts were subjected to NF- $\kappa$ B-specific EMSA. Specificity of the NF- $\kappa$ B and DNA-binding reaction was confirmed by incubation of liver nuclear extracts with increasing concentration of unlabeled ('cold') oligonucleotides for NF- $\kappa$ B and anti-p50 antibody. (b) Total liver protein extracts were probed with anti-phospho (p)-STAT1 (Tyr-701), anti-STAT1, anti-phospho (p)-STAT3 (Tyr-705) and anti-STAT3 antibodies and subjected to immunoblot analysis; (c) the ratio of band intensity was quantified and calculated by Biorad-Image for Windows Program (BioRad GS-800 Calibrated Densitometer). Results are shown as mean \pm s.d. Data are representative of five experiments. ${ }^{* * *} P<0.001$.

cytokines is followed by activation of chemotactic factors such as IP-10, MCP-1 and MIP- $1 \alpha$, which attract more leukocytes to the liver to amplify local inflammation and damage. ${ }^{31,32}$ IP-10 plays an important role in hepatic neutrophil influx in $\mathrm{CIH}^{31}$ Although $\mathrm{CIH}$ is developing, IP-10 expression has been found to increase. ${ }^{33}$ In MIP- $1 \alpha-/-$ mice, CIH has been shown to be markedly decreased, indicating that MIP- $1 \alpha$ is significantly involved in the pathogenesis of the disease. ${ }^{34}$ Our observation of reduced production of all the above inflammatory molecules in SCD1-deficient mice after $\mathrm{CIH}$ induction suggests that the absence of SCD1 abrogates immune-induced hepatocytic injury and inflammation cascade. In other words, in the setting of T-cell-mediated liver injury, increased SCD1 expression and lipid accumulation may promote proinflammatory cytokine production.

Next, we explored further whether key pathogenic signaling pathways in $\mathrm{CIH}$ were altered in SCD1-deficient mice.
Because transcription factor NF- $\kappa \mathrm{B}$ is involved in the synthesis of TNF- $\alpha$, IFN- $\gamma$ and numerous proinflammatory factors, the role of NF- $\kappa \mathrm{B}$ in the pathogenesis of $\mathrm{CIH}$ has been well recognized. Many drugs show significant protective effects against $\mathrm{CIH}$ depending upon their prevention of NF$\kappa \mathrm{B}$ activation. $^{35,36}$ In SCD1-deficient mice, DNA-binding activity of NF- $\kappa \mathrm{B}$ after ConA administration was much weaker as indicated by EMSA. Another key pathogenic signaling pathway involved in CIH is IFN- $\gamma /$ STAT1 signaling. Liver injury induced by ConA was associated with an increase in pSTAT1 levels in the liver. Transgenic mice overexpressing STAT1 under the control of the CD2 promoter enhancer construct showed elevated IFN- $\gamma$ levels as well as significantly augmented liver injury following administration of ConA. Consistently, STAT1-/- mice were protected from such T-cell-dependent liver injury. ${ }^{25}$ Here, we observed significantly reduced phosphorylation of STAT1 with ConA treatment in SCD1-deficient mice. These results suggest that 

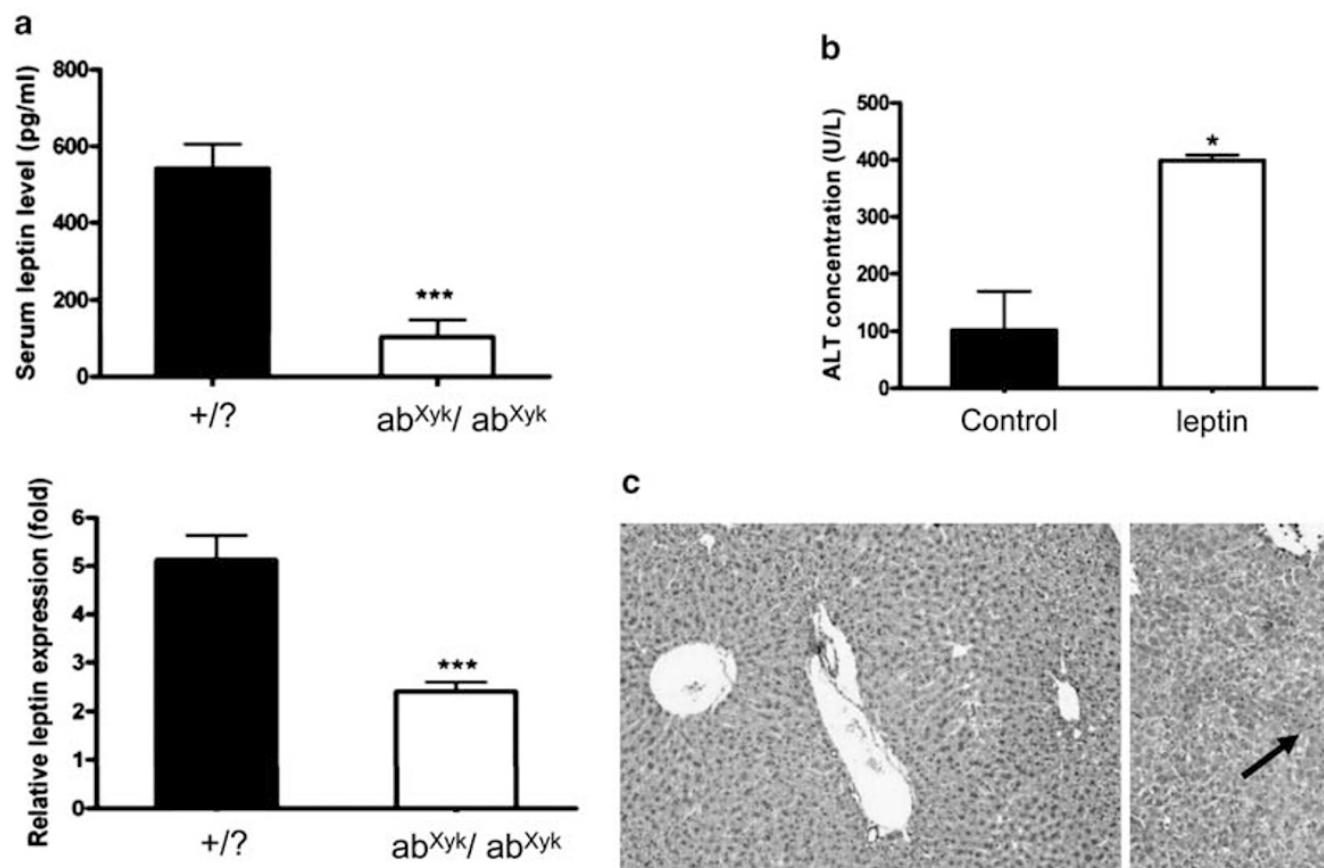

C
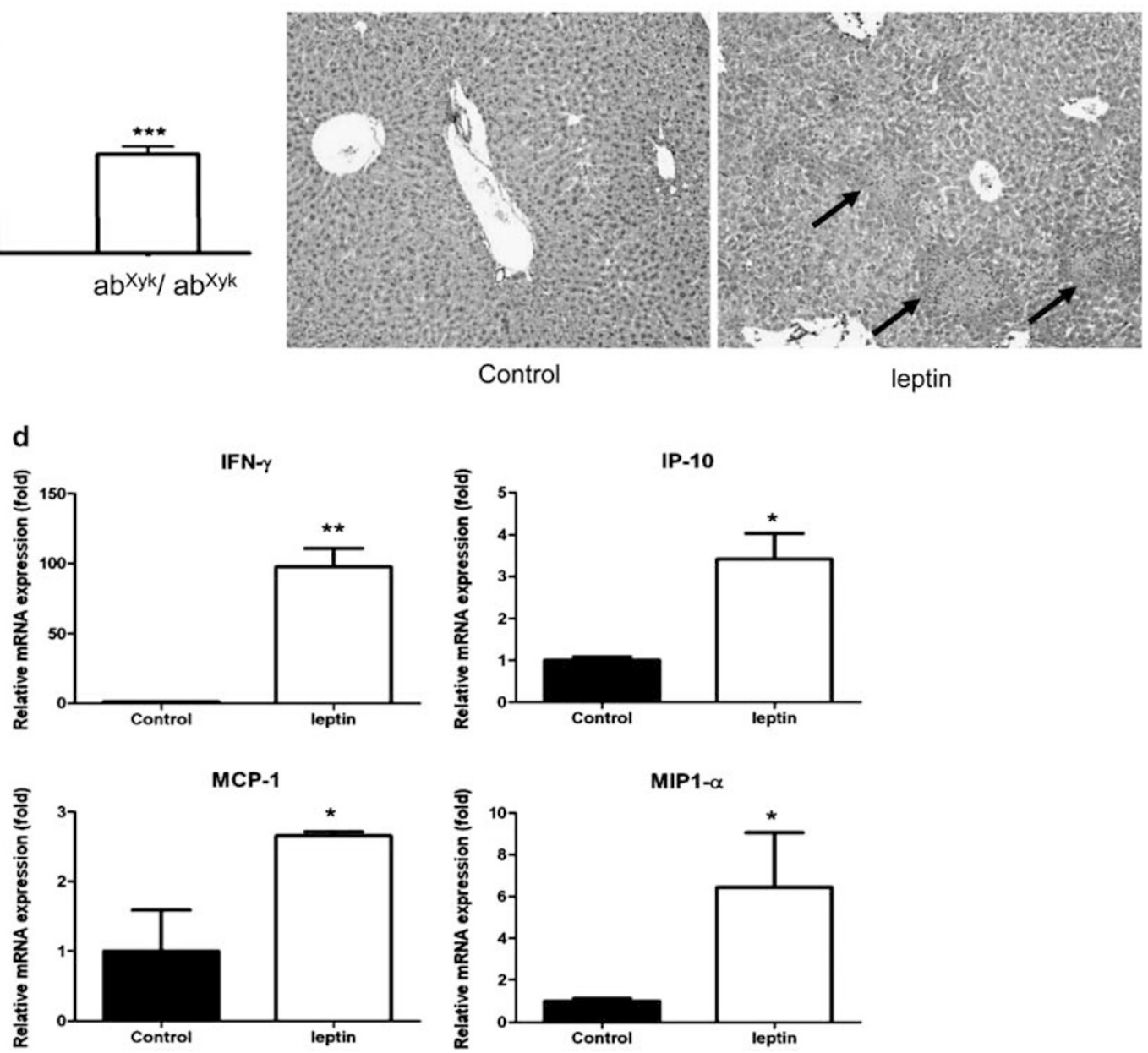

Figure 5 Leptin mediated the resistance to $\mathrm{CIH}$ in SCD1-deficient mice. (a) Plasma leptin levels of $+/$ ? and ab $\mathrm{b}^{\mathrm{Xyk}} / \mathrm{ab}^{\mathrm{Xyk}}$ mice were measured with ELISA. mRNA expression of leptin in epididymal fat pad was quantitatively analyzed using real-time PCR. Results are shown as mean $\pm s . d$. ( $n=5)$. (b) ab ${ }^{x y k} / a b^{x y k}$ mice received i.p. injections of either PBS or leptin $(1 \mathrm{mg} / \mathrm{kg})$ twice a day for 5 days. Subsequently, CIH was induced in all mice. Plasma ALT levels were measured $17 \mathrm{~h}$ later. (c) Representative liver H\&E staining of (b). Arrows indicates necrotic areas. (d) Liver mRNA expression of IFN- $\gamma$, IP-10, MCP-1 and MIP-1 $\alpha$ were quantitatively analyzed using real-time PCR. Results are shown as mean \pm s.d. $(n=5)$. Data are representative of three experiments. ${ }^{\star} P<0.05$, ${ }^{* *} P<0.01,{ }^{* * *} P<0.001$.

SCD1 deficiency impairs the production of IFN- $\gamma$, thus reducing the activation STAT1 in $\mathrm{CIH}$, and protecting mice from immune-mediated liver injury. In addition, we tested the STAT3 pathway which mediates protective effects in $\mathrm{CIH}^{37}$ We showed that the STAT3 pathway was not significantly influenced in SCD1-deficient mice.
We wondered how SCD1, the enzyme for biosynthesis of MUFAs, might interfere with inflammation. We noticed a report by Ntambi et al that leptin production was suppressed in SCD1-deficient mice on chow and high-fat diet. ${ }^{14}$ It is well known that leptin is a critical linker between energy metabolism and immunity. ${ }^{13}$ Leptin influences many immune 
pathways in a direct or indirect manner. The key pathogenic immune cells in $\mathrm{CIH}$, such as T cells and NKT cells, may be activated by leptin. ${ }^{13}$ Leptin also promotes the production of proinflammatory cytokines such as TNF- $\alpha$ and IFN- $\gamma$ which are critical in the pathogenesis of $\mathrm{CIH} .{ }^{13,38}$ A previous study showed that susceptibility to $\mathrm{CIH}$ may be increased by leptin via maintenance of high numbers of hepatic NKT cells as well as by mediation of T-cell activation and cytokine production. ${ }^{14}$ Obesity and the metabolic syndrome would therefore put the liver at a heightened risk of immune-mediated damage under a leptin-dependent mechanism. ${ }^{39}$ Leptin deficiency leads to reduced severity in the $\mathrm{CIH}$ model. ${ }^{15}$ Here, we observed reduced serum leptin levels and leptin mRNA expression in adipose tissue in SCD1-deficient mice. The replacement experiment further confirmed that reduced leptin levels contributed significantly to the protective role of SCD1 deficiency in CIH induction.

Very recently, several well-known lipid metabolism regulators that stimulate fat oxidation, including metformin and Omega-3 polyunsaturated fatty acids, have shown hepatoprotective activity against immune-mediated liver injury. ${ }^{40,41}$ In this study, we clearly demonstrated that inactivation of SCD1, a key regulator in lipid metabolism, protects mice from $\mathrm{CIH}$. Similar protective effects were also seen after pretreatment of mice with AMPK activator metformin (our unpublished data). Our findings provide direct evidence that regulation of energy metabolism may alter immune responses in the liver, a realization that suggests a new approach to understanding and perhaps controlling crosstalk between energy metabolism and the immune system in the liver. Such an approach may assist and improve liver disease treatment in the future.

\section{ACKNOWLEDGEMENT}

This work was supported by the National Foundation of Natural Science (30671994) and the Knowledge Innovation Program of the Chinese Academy of Sciences (J0171-1905).

1. Wellen KE, Hotamisligil GS. Inflammation, stress, and diabetes. J Clin Invest 2005;115:1111-1119.

2. Gao B, Jeong WI, Tian Z. Liver: an organ with predominant innate immunity. Hepatology 2008;47:729-736.

3. Racanelli $\mathrm{V}$, Rehermann B. The liver as an immunological organ. Hepatology 2006;43:S54-S62.

4. Guebre-Xabier M, Yang S, Lin HZ, et al. Altered hepatic lymphocyte subpopulations in obesity-related murine fatty livers: potential mechanism for sensitization to liver damage. Hepatology 2000;31: 633-640.

5. Li Z, Soloski MJ, Diehl AM. Dietary factors alter hepatic innate immune system in mice with nonalcoholic fatty liver disease. Hepatology 2005;42:880-885.

6. Kremer M, Hines IN, Milton RJ, et al. Favored T helper 1 response in a mouse model of hepatosteatosis is associated with enhanced $T$ cellmediated hepatitis. Hepatology 2006;44:216-227.

7. Lu Y, Bu L, Zhou S, et al. Scd1ab-Xyk: a new asebia allele characterized by a CCC trinucleotide insertion in exon 5 of the stearoyl-CoA desaturase 1 gene in mouse. Mol Genet Genomics 2004;272: 129-137.
8. Nagaki M, Naiki T, Brenner DA, et al. Tumor necrosis factor alpha prevents tumor necrosis factor receptor-mediated mouse hepatocyte apoptosis, but not fas-mediated apoptosis: role of nuclear factorkappaB. Hepatology 2000;32:1272-1279.

9. Cohen P, Ntambi JM, Friedman JM. Stearoyl-CoA desaturase-1 and the metabolic syndrome. Curr Drug Targets Immune Endocr Metabol Disord 2003;3:271-280.

10. Dobrzyn A, Ntambi JM. The role of stearoyl-CoA desaturase in body weight regulation. Trends Cardiovasc Med 2004;14: 77-81.

11. Zheng $\mathrm{Y}$, Eilertsen $\mathrm{KJ}, \mathrm{Ge} \mathrm{L}$, et al. Scd1 is expressed in sebaceous glands and is disrupted in the asebia mouse. Nat Genet 1999;23: 268-270.

12. Miyazaki M, Kim YC, Gray-Keller MP, et al. The biosynthesis of hepatic cholesterol esters and triglycerides is impaired in mice with a disruption of the gene for stearoyl-CoA desaturase 1. J Biol Chem 2000;275:30132-30138.

13. La Cava A, Matarese G. The weight of leptin in immunity. Nat Rev Immunol 2004;4:371-379.

14. Ntambi JM, Miyazaki M, Stoehr JP, et al. Loss of stearoyl-CoA desaturase-1 function protects mice against adiposity. Proc Natl Acad Sci USA 2002;99:11482-11486.

15. Faggioni R, Jones-Carson J, Reed DA, et al. Leptin-deficient (ob/ob) mice are protected from $T$ cell-mediated hepatotoxicity: role of tumor necrosis factor alpha and IL-18. Proc Natl Acad Sci USA 2000;97:2367-2372.

16. Ma X, Hua J, Mohamood AR, et al. A high-fat diet and regulatory T cells influence susceptibility to endotoxin-induced liver injury. Hepatology 2007:46:1519-1529.

17. Kim KH, Shin $\mathrm{HJ}$, Kim K, et al. Hepatitis B virus $X$ protein induces hepatic steatosis via transcriptional activation of SREBP1 and PPARgamma. Gastroenterology 2007;132:1955-1967.

18. Endo M, Masaki T, Seike M, et al. TNF-alpha induces hepatic steatosis in mice by enhancing gene expression of sterol regulatory element binding protein-1c (SREBP-1c). Exp Biol Med (Maywood) 2007;232: 614-621.

19. Bergheim I, Guo L, Davis MA, et al. Metformin prevents alcoholinduced liver injury in the mouse: critical role of plasminogen activator inhibitor-1. Gastroenterology 2006;130:2099-2112.

20. Enoch HG, Catala A, Strittmatter P. Mechanism of rat liver microsomal stearyl-CoA desaturase. Studies of the substrate specificity, enzymesubstrate interactions, and the function of lipid. J Biol Chem 1976;251:5095-5103.

21. Chen C, Shah YM, Morimura K, et al. Metabolomics reveals that hepatic stearoyl-CoA desaturase 1 downregulation exacerbates inflammation and acute colitis. Cell Metab 2008;7:135-147.

22. Sass $G$, Heinlein $S$, Agli $A$, et al. Cytokine expression in three mouse models of experimental hepatitis. Cytokine 2002;19: $115-120$.

23. Mizuhara $\mathrm{H}, \mathrm{O}$ 'Neill E, Seki N, et al. T cell activation-associated hepatic injury: mediation by tumor necrosis factors and protection by interleukin 6. J Exp Med 1994;179:1529-1537.

24. Kusters $S$, Gantner F, Kunstle $G$, et al. Interferon gamma plays a critical role in T cell-dependent liver injury in mice initiated by concanavalin $A$. Gastroenterology 1996;111:462-471.

25. Siebler J, Wirtz S, Klein S, et al. A key pathogenic role for the STAT1/Tbet signaling pathway in T-cell-mediated liver inflammation. Hepatology 2003;38:1573-1580.

26. Neish AS, Read MA, Thanos $D$, et al. Endothelial interferon regulatory factor 1 cooperates with NF-kappa B as a transcriptional activator of vascular cell adhesion molecule 1. Mol Cell Biol 1995;15: 2558-2569.

27. Yoneda $\mathrm{M}$, Wada $\mathrm{K}$, Katayama $\mathrm{K}$, et al. A novel therapy for acute hepatitis utilizing dehydroepiandrosterone in the murine model of hepatitis. Biochem Pharmacol 2004;68:2283-2289.

28. Sass $G$, Koerber K, Bang $R$, et al. Inducible nitric oxide synthase is critical for immune-mediated liver injury in mice. J Clin Invest 2001;107:439-447.

29. Okamoto T, Masuda $Y$, Kawasaki T, et al. Aminoguanidine prevents concanavalin A-induced hepatitis in mice. Eur J Pharmacol 2000;396:125-130

30. Marshall HE, Merchant K, Stamler JS. Nitrosation and oxidation in the regulation of gene expression. FASEB J 2000;14:1889-1900. 
31. Tamaru M, Nishioji K, Kobayashi Y, et al. Liver-infiltrating T lymphocytes are attracted selectively by IFN-inducible protein-10. Cytokine 2000;12:299-308.

32. Nakamura K, Okada M, Yoneda $M$, et al. Macrophage inflammatory protein-2 induced by TNF-alpha plays a pivotal role in concanavalin Ainduced liver injury in mice. J Hepatol 2001;35:217-224.

33. Jaruga $\mathrm{B}$, Hong $\mathrm{F}$, Kim WH, et al. IFN-gamma/STAT1 acts as a proinflammatory signal in T cell-mediated hepatitis via induction of multiple chemokines and adhesion molecules: a critical role of RF-1. Am J Physiol Gastrointest Liver Physiol 2004;287: G1044-G1052.

34. Ajuebor MN, Hogaboam CM, Le T, et al. CCL3/MIP-1alpha is pro-inflammatory in murine $T$ cell-mediated hepatitis by recruiting CCR1-expressing CD4(+) T cells to the liver. Eur J Immunol 2004;34:2907-2918.

35. Imose $M$, Nagaki $M$, Kimura $K$, et al. Leflunomide protects from T-cellmediated liver injury in mice through inhibition of nuclear factor kappaB. Hepatology 2004;40:1160-1169.
36. Schumann J, Prockl J, Kiemer AK, et al. Silibinin protects mice from T cell-dependent liver injury. J Hepatol 2003;39: 333-340.

37. Hong F, Jaruga B, Kim WH, et al. Opposing roles of STAT1 and STAT3 in T cell-mediated hepatitis: regulation by SOCS. J Clin Invest 2002;110:1503-1513.

38. Matarese G, Moschos S, Mantzoros CS. Leptin in immunology. J Immunol 2005;174:3137-3142.

39. Sennello JA, Fayad R, Morris AM, et al. Regulation of T cell-mediated hepatic inflammation by adiponectin and leptin. Endocrinology 2005;146:2157-2164.

40. Schmocker C, Weylandt $\mathrm{KH}$, Kahlke L, et al. Omega-3 fatty acids alleviate chemically induced acute hepatitis by suppression of cytokines. Hepatology 2007;45:864-869.

41. Bergheim I, Luyendyk JP, Steele C, et al. Metformin prevents endotoxin-induced liver injury after partial hepatectomy. J Pharmacol Exp Ther 2006;316:1053-1061. 